\title{
Probing the Gas Distribution of Ly $\alpha$ Emitting Galaxies through their Spectrally Resolved Ly $\alpha$ Emission
}

\section{Taylor S. Chonis*}

Department of Astronomy, University of Texas at Austin

E-mail: tschoniseastro.as.utexas.edu

\section{Guillermo A. Blanc}

Observatories of the Carnegie Institution for Science

E-mail: gblancm@obs. carnegiescience. edu

\section{Gary J. Hill}

McDonald Observatory, University of Texas at Austin

E-mail: hilleastro.as.utexas.edu

\begin{abstract}
We present the preliminary results of Magellan/IMACS deep, multi-object spectroscopic observations of 25 Ly $\alpha$ emitting galaxies (LAEs) at $2 \lesssim z \lesssim 3.5$ that were drawn primarily from the HETDEX Pilot Survey in the COSMOS field. With a spectral resolution of $150 \mathrm{~km} \mathrm{~s}^{-1}$ FWHM, we resolve the $\operatorname{Ly} \alpha$ emission line profiles of these galaxies and observe an assortment of velocity and spatial structure due to the resonant scattering of Ly $\alpha$ photons in the H I gas of the galaxies' interstellar and circumgalactic media. The spectral structure includes varying degrees of asymmetry, a range of line widths, and a varying number of emission peaks with different velocity separations. Through parametric fitting of the Ly $\alpha$ line profiles, we quantify the observed structure and find evidence of a correlation between parameters describing the shape of the strongest emission line component and its velocity offset from $z_{\text {sys }}$, the galaxy's systemic redshift. Recent simulations have predicted a similar trend that is driven by the neutral hydrogen column density $N_{\mathrm{HI}}$ as a general property of Ly $\alpha$ radiative transfer in various H I distributions and kinematic configurations.
\end{abstract}

Frank N. Bash Symposium 2013: New Horizons in Astronomy

October 6-8, 2013

Austin, Texas

\footnotetext{
*Speaker.
} 

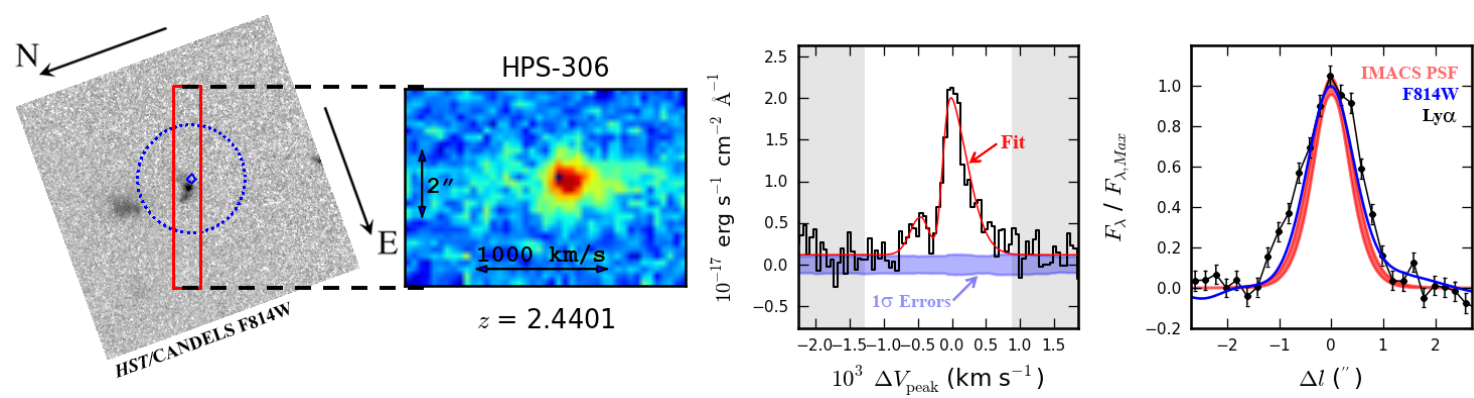

Figure 1: IMACS spectra of HPS-306, a double-peaked and extended LAE. Left: HST F814W image. The IMACS slit is shown in red. The blue diamond (circle) is the Ly $\alpha$ centroid (positional error) from [2]. Middle Left: IMACS 2D spectrum. Middle Right: IMACS 1D spectrum plotted in velocity relative to the

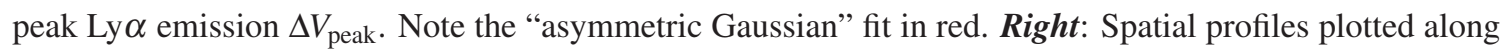
the length of the slit $\Delta l$ for the Ly $\alpha$ and continuum emission relative to the IMACS PSF.

\section{Background and Observations}

Ly $\alpha$ photons $(1215 \AA$ ) are produced in the H II regions of star-forming galaxies due to hydrogen recombination after ionization by the UV radiation of young stars. The resonant scattering nature of the Ly $\alpha$ transition in $\mathrm{H}$ I results in frequency and spatial diffusion as photons random-walk out of the galaxy. This makes $\operatorname{Ly} \alpha$ radiative transfer dependent on the distribution and kinematics of the H I gas, as well as the galaxy's dust content. The shape of the emergent Ly $\alpha$ spectrum thus encodes the physics of the scattering gas in a non-trivial way (e.g., [1]). We attempt to obtain a global perspective of the processes governing the escape of Ly $\alpha$ by comparing observed trends among the Ly $\alpha$ emission properties for a large sample of LAEs. We have obtained multi-slit Ly $\alpha$ spectra with Magellan/IMACS of 25 LAEs at $2 \lesssim z \lesssim 3.5$ in COSMOS, drawn from the HETDEX Pilot Survey [2,3] and a narrowband survey for LAEs at $z \approx 2.25$ [4]. The spectra have $150 \mathrm{~km}$ $\mathrm{s}^{-1}$ FWHM spectral resolution, 0.9" FWHM spatial resolution along the slit (7.3 kpc at $z=2.75$ ), and a $1 \mathrm{D} 5 \sigma$ line flux limit of $2 \times 10^{-18} \mathrm{erg} \mathrm{s}^{-1} \mathrm{~cm}^{-2}$ in 5.25 hours of exposure at $4500 \AA$.

\section{Ly $\alpha$ Classification and Spectral Line Profile Fitting}

Fig. 1 shows an example of our IMACS data. Excluding two broad-line AGN, we classify the remaining 23 LAEs into three categories based on the 1D Ly $\alpha$ spectral line profiles: single peaks (48\% of the sample), double peaks (43\%), and LAEs with greater than two Ly $\alpha$ peaks $(9 \%)$. These results are consistent with previous work [5]. The Ly $\alpha$ spectra are also collapsed spectrally to yield a spatial profile along the slit. To compare the spatial Ly $\alpha$ extent to that of the continuum, we convolve COSMOS F814W HST images ( $2200 \AA$ in the rest-frame) with the IMACS PSF and collapse the resulting image along the width of the slit. We find five LAEs with Ly $\alpha$ emission that is extended significantly beyond that of the continuum. Six LAEs are resolved in Ly $\alpha$ and continuum, while the majority (11 LAEs) are completely unresolved. Although our sample is small when subdivided in this way, we find no significant connection between the Ly $\alpha$ spectral classification and whether or not an LAE displays extended Ly $\alpha$ emission.

To quantify the spectral morphology of the Ly $\alpha$ line, we fit an "asymmetric Gaussian" function [6] to the 1D data, including an instrumental correction. For the double-peaked Ly $\alpha$ subsample, 

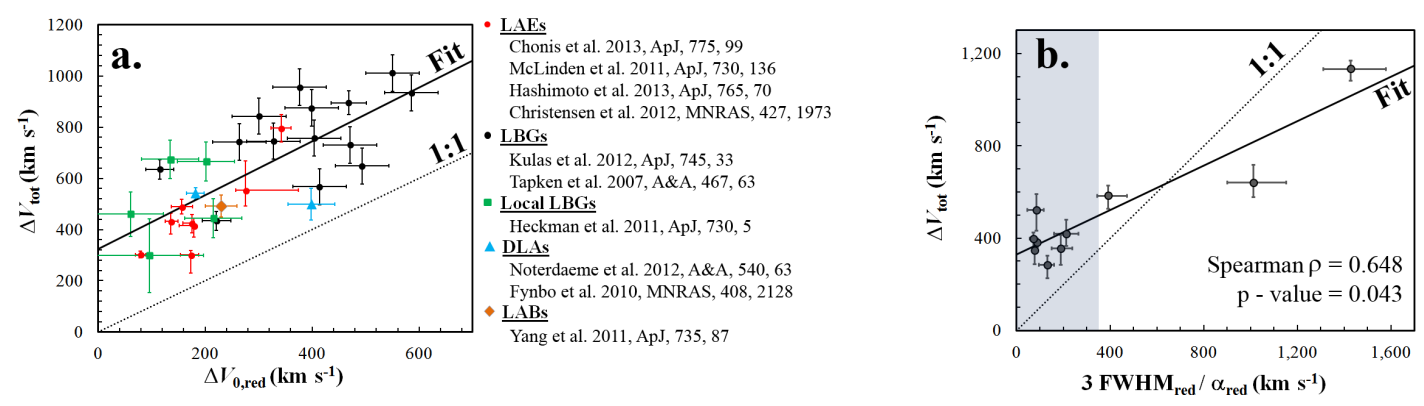

Figure 2: $\boldsymbol{a})$ The relation between $\Delta V_{\text {tot }}$ and $\Delta V_{0, \text { red }}$ for double-peaked Ly $\alpha$ showing that $\Delta V_{\text {tot }}$ scales with $\Delta V_{0, \text { red }}$ on average (but is offset from the unity relation). The data was compiled from the sources listed to the right of the plot. $\boldsymbol{b}$ ) The correlation between $\Delta V_{\text {tot }}$ and $3 \mathrm{FWHM}_{\mathrm{red}} / \alpha_{\mathrm{red}}$, as derived from our Ly $\alpha$ line profile fitting for the double-peaked subsample, similar to the theoretical result shown in Fig. 16 of [7]. The blue region corresponds to the range of $3 \mathrm{FWHM}_{\text {red }} / \alpha_{\text {red }}$ covered in that work. Measurements of $z_{\text {sys }}$ may result in a reduction of the scatter in the ordinate axis by allowing us to plot $\Delta V_{0, \text { red }}$ directly rather than $\Delta V_{\text {tot }}$.

the measured parameters that are relevant here are the peak-to-peak velocity separation $\Delta V_{\text {tot }}$, and the width $\left(\mathrm{FWHM}_{\mathrm{red}}\right)$ and asymmetry $\left(\alpha_{\text {red }}\right.$; defined in [6]) of the strongest emission component (i.e., the red peak for our sample). Currently, we do not have measurements of $z_{\text {sys }}$ for these LAEs, so the $\operatorname{Ly} \alpha$ velocity zero-point is unknown. However, data compiled from the literature shows that $\Delta V_{\text {tot }}$ scales with the absolute velocity offset of the red Ly $\alpha$ peak $\Delta V_{0, \text { red }}$ (see Fig. $2 a$ ), albeit with significant scatter. With this in mind, we find a correlation between $\Delta V_{\text {tot }}$ and $3 \mathrm{FWHM}_{\text {red }} / \alpha_{\text {red }}$, a parameter defined by [7] that describes the shape of the strongest Ly $\alpha$ emission peak (see Fig. 2b).

According to radiative transfer simulations by [7], LAEs with large $\Delta V_{0 \text {,red }}$ (here, $\Delta V_{\text {tot }}$ ) and large $3 \mathrm{FWHM}_{\mathrm{red}} / \alpha_{\text {red }}$ have high $N_{\mathrm{HI}}$. Deviations from this correlation can indicate different kinematic/spatial configurations of the $\mathrm{H}$ I gas. If the observed relation between $3 \mathrm{FWHM}_{\text {red }} / \alpha_{\text {red }}$ and $\Delta V_{0, \text { red }}$ is sufficiently tight, it can also be used to predict $z_{\text {sys }}$ based on observations of only Ly $\alpha$. This could be powerful for studies that employ spectral stacking techniques to probe the UV continuum of LAEs. To properly explore this, we plan to obtain $z_{\text {sys }}$ for our sample by observing their non-resonant nebular emission lines (e.g., [O III] $\lambda$ 5007), which may reduce the scatter in Fig. $2 b$ by allowing us to plot $\Delta V_{0 \text {,red }}$ directly. Additional observations of Ly $\alpha$, particularly for LAEs with large $\Delta V_{\text {tot }}$ (i.e., $\Delta V_{0 \text {,red }}$ ) and $3 \mathrm{FWHM}_{\text {red }} / \alpha_{\text {red }}$, will also help to increase the statistical significance of the correlation shown in Fig. $2 b$. This work will be featured in a future unabridged publication.

\section{References}

[1] A. Verhamme, D. Schaerer, \& A. Maselli, 2006, $A \& A, 460,397$

[2] J.J. Adams, G.A. Blanc, G.J. Hill, et al., 2011, ApJS, 192, 5

[3] G.A. Blanc, J.J. Adams, K. Gebhardt, et al., 2011, ApJ, 736, 31

[4] K.K. Nilsson, C. Tapken, P. Møller, et al., 2009, A\&A, 498, 13

[5] T. Yamada, Y. Matsuda, K. Kousai, et al., 2012, ApJ, 751, 29

[6] T.S. Chonis, G.A. Blanc, G.J. Hill, et al., 2013, ApJ, 775, 99

[7] Z. Zheng \& J. Wallace, 2013, ApJ submitted, arXiv:1308.1405 